\title{
TELEREHABILITATION OF THE KNEE JOINTS OF PATIENTS WITH POLYTRAUMA
}

10.36740/WLek202101109

\author{
Andriy I. Tsvyakh, Andriy Y. Hospodarskyy, Nataliia 0. Marchenkova, Ihor R. Kopytchak, Volodymyr P. Kostjuk, \\ Yevhen A. Lymar, Sergii M. Gdanskyi \\ I. HORBACHEVSKYY TERNOPIL NATIONAL MEDICAL UNIVERSITY, TERNOPIL, UKRAINE
}

\begin{abstract}
The aim: The overarching theme of this paper is to discuss implementation of the telemedicine technology for rehabilitation of patients with injuries of the lower extremities. Materials and methods: Consecutive patients were recruited over a four-year period. A total of 48 polytrauma patients with lower extremity injuries were enrolled in the study after the resolution of the surgical sequelae. 16 patients from the control group underwent traditional rehabilitation procedures for a 3-week period after injury. A total of 32 subjects were enrolled in the telerehabilitation group for a 3-week study period after injury and were trained with a set of exercises for home use. Home remote monitoring for the 96 test subjects included use of a Portable device with Axis-sensor, temperature and volume sensors, that were fixed to the injured limb. Software permits the monitoring of exercise time, local temperature, the biomechanics of active movements of the injured limb.

Results: The orthopedic surgeon during telerehabilitation took significantly less time to consult patients (1.9 minutes) than the traditional rehabilitation (15.2 minutes). Patient satisfaction was higher for the telerehabilitation with machine learning algorithm (78.3\%) than for the orthopedic surgeon's traditional rehabilitation (36.7\%).

Conclusions: Subjects reported a higher satisfaction with telerehabilitation than with the traditional orthopedic rehabilitation due to the fact that they spent less time at the hospital and had more time for exercises at home under orthopedic remote monitoring. The telerehabilitation system can be used in complex rehabilitation of patients with injuries of the lower extremities.
\end{abstract}

KEY WORDS: telemedicine, rehabilitation, polytrauma

\section{INTRODUCTION}

The continuous development of technology that paves the way towards the expansion of connections through the internet and the growth of the capacity to process data has created greater possibilities of the development of the global health industry especially telemedicine [1]. Telemedicine is the application of transferring medical information in through digital communication to perform consultations, medical examinations and rehabilitation procedures. The main objectives of telemedicine are bridging the gap of accessibility and communication in the medical field reducing delays and the cost logistics [2]. Wireless technology applied to sensors and application to case studies related to home monitoring and have been developing during the past decade, including studies on the cost-effectiveness [3]. Polytrauma patients have numerous risk factors. Early assessment of the clinical status of patients with polytrauma is of pivotal importance in guiding surgical and future rehabilitation [4].

The use of a device such that a person may wear, may allow for constant monitoring of a patient and for the ability to notice changes that may be less distinguishable by humans [5].

Timely access to orthopedic rehabilitation is an important problem for the world health care system. Presently, wait times to see an orthopedic surgeon can exceed two months and with the rapidly aging population as well as the increased incidence of obesity, the need for orthopedic care is expected to drastically increase in the coming years. Efforts have been made to ensure timely access to orthopedic care for the population yet, despite an investment in resources, wait times for a consult remain excessively long [6].

Therefore, the overarching theme of this paper is to acknowledge the gold standard in implementation of the telemedicine technology for rehabilitation of the patients with injuries of the lower extremities. The main prerequisite for success within the orthopedic community is to identify indications and contraindications for distance rehabilitation [7]. Lower Extremity Functional Scale is widely used to evaluate the functional disabilities of a patient with a lower extremity disorder. The LEFS score ranges from 0 to 80 with a higher score indicating a higher functional status. The use of the LEFS in research studies has been validated and the LEFS is a reliable tool for assessing lower extremity functional status [8].

An application telemedicine for monitoring and gathering data on a patient's progress without the use of video communication or consultation is the study on joints Rehabilitation System with Feedback from the Wireless Prototype [9-11]. 


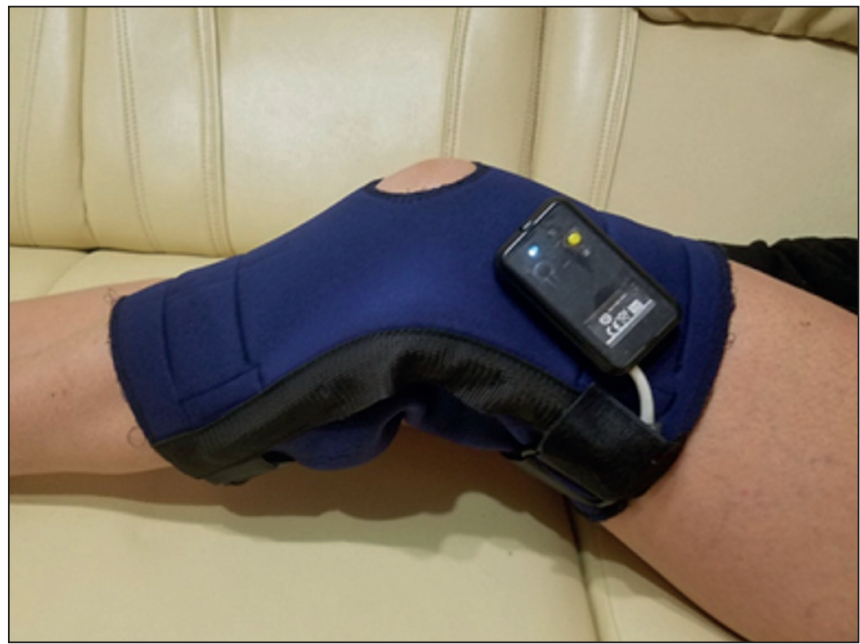

Fig. 1. Portable device with sensors.

Joints rehabilitation system connected through internet to record the number of use and effects of telerehabilitation therapy and using measure the efficacy of the rehabilitation strategy. This is an example of the importance of wireless vital sensors to telemedicine with its capacity to easily transmit data to devices which is vital in continuous monitoring. Through the current developments in patient monitoring, there is a support for self-diagnosing techniques and application of telemedicine not only in hospitals but also in homes [12]. As the capacity expands with telemedicine being applied on devices outside the hospitals, each device should be designed with a standard data architecture.

\section{THE AIM}

The overarching theme of this paper is to discuss implementation of the telemedicine technology for rehabilitation of patients with injuries of the lower extremities.

\section{MATERIALS AND METHODS}

Consecutive polytrauma patients were recruited after recovery over a five-year period - September 2018 to February 2020 - from the waiting lists of the department of orthopedic surgery, at Ternopil Emergency Hospital that serves as a trauma center. A total of 48 polytrauma patients with lower extremity injuries were enrolled in the study after the resolution of the surgical sequelae and monitored during a 3-weeks period.

All enrolled subjects signed a consent form prior to participating in the study. The Research Ethics Board of the I. Horbachevskyy Ternopil Medical University, Ukraine approved the research study.

16 patients from the control group underwent traditional rehabilitation procedures for a 3-weeks period after injury. This usual care group included the generally accepted methods of rehabilitation therapy (i.e. massage, myostimulation, walking without a load on the injured limb, and exercises in the pool).
A total of 32 subjects were enrolled in the telerehabilitation group for a 3-week study period after injury and were trained with a set of exercises for home use.

Home remote monitoring for the 32 test subjects included use of a Portable device (Fig 1.) with Axis-sensor, temperature, volume and pulse sensors, that were fixed to the injured limb. Software permits the monitoring of biomechanical movement time, local temperature, the frequency of active movements and volume of the injured limb. During the execution of home exercises, data from the subjects Portable device were measured and sent to a server through a cellular Internet connection and to the personal smartphone of the rehabilitation doctor and displayed as digital data and graphically.

The Tele-rehabilitation protocol included:

- Fixation of the Portable device to the injured limb and use of customized software.

- Home exercises: Passive flexion-extension in the leg. - Home exercises: Active flexion-extension in the leg.

Patients were also trained for subjective assessment of pain on a 10 -point scale. The initial implementation of this telerehabilitation model did not include prescription of medications or joint injections.

The software contained a tailored personal rehabilitation record for health information and communication between the patient and health professionals. Basic measures for patients included blood pressure, volume of limb, waist, chest, pulse, weight, height, sex, saturation, local temperature, movement activity and condition of connective tissue.

Subjects completed a questionnaire where they provided anthropometric data as well as information on their education, employment, household income, household living status, and information on clinical variables such as the joints effected, the reason for consulting, the duration of their symptoms, the use of a walking aid and the presence of any co-morbidities. All subjects from both groups also completed the Lower Extremity Functional Scale (LEFS) questionnaire. The majority of patients $(75 \%)$ cited pain as the reason for consult with the orthopedic surgeon and all of the patients (100\%) consulted for a knee disorder (after injuries of the lower extremities). All patients had imaging tests available in their file at the time of consult (X-Ray and CT-scan).

Based on the patient's individual condition, the rehabilitation doctor created an individualized rehabilitation plan for each subject, containing an activity plan. All patients had personal goals for daily movement activity and steps in the rehabilitation program.

All subjects from telerehabilitation group were assigned a personal doctor. The control group received traditional rehabilitation at home without a personal doctor.

\section{RESULTS AND DISCUSSION}

In the telerehabilitation arm of the study, 48 subjects with injuries of the lower extremities were symptomatic for at least 3 weeks.

Subjects from telerehabilitation group had a mean age of 44.7 years and the majority were men (67.3\%). The mean 


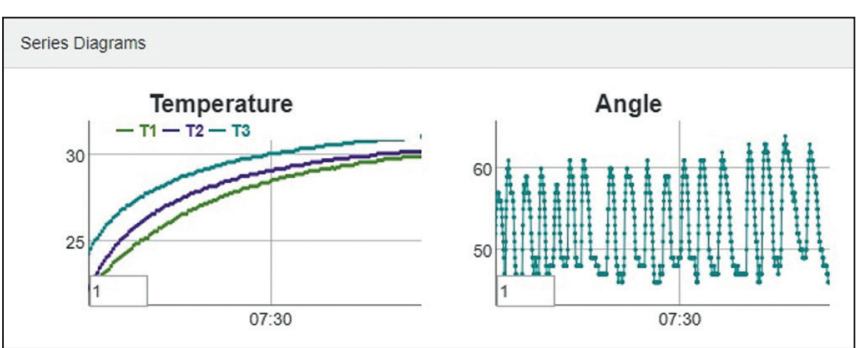

Fig. 2. First day of telerehabilitation.

Table 1. Characteristics of the study participants of the telerehabilitation group $(\mathrm{n}=32)$

\begin{tabular}{ccc}
\hline Characteristics & $\mathbf{n ~ ( \% )}$ & Mean (SD) \\
\hline Age (years) & & $44.7(5.4)$ \\
\hline Gender & \\
\hline Male & $21(65.6 \%)$ \\
\hline Female & $27(34.4 \%)$ \\
\hline Body mass index $\left(\mathrm{kg} / \mathrm{m}^{2}\right)$ & & $27.6(3.6)$ \\
\hline Employment & \\
\hline Employed & $17(35.4 \%)$ \\
\hline Unemployed & $15(31.2 \%)$ \\
\hline Retired & - & \\
\hline Duration of symptoms (days) & $14(3)$ \\
\hline Patients using walking aid & $39(81.3 \%)$ \\
\hline Lower Extremity Functional Scale \\
- LEFS score (\%)
\end{tabular}

Lower Extremity Functional Scale (LEFS) score of patients was 44.62. Table 1 presents selected characteristics of the participants of the telerehabilitation group.

In the control group of the study, 16 subjects with injuries of the lower extremities were symptomatic for at least 3 weeks and continued rehabilitation at home without an assigned doctor. Subjects had a mean age of 48.6 years and the majority were men (65.4\%). The mean Lower Extremity Functional Scale (LEFS) score of patients was 38.31.

During the telemonitoring, the physician controls the adequacy of execution of each stage of rehabilitation exercises and has the ability to adjust the load in real time depending on the functional state of the limb (Fig 2, Fig. 3.).

Subjects were also asked if their pain level increased after the first exercise and in the event that it did, they were asked to indicate by how much it increased by picking one of the following three options on the smartphone: 1-4 pain was a bit stronger; 5 -7- pain was moderately stronger; 8 -10 pain was much stronger. Software allows increasing the daily load, if the assessment of pain after exercise was not more

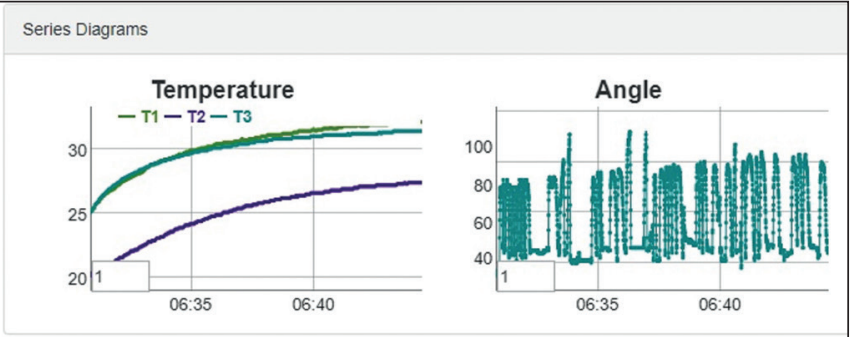

Fig. 3. 14th day of telerehabilitation.

than 7 points on 10-point scale and progressive limb edema was absent. If pain persisted or questions persisted, there was correction of the rehabilitation algorithm by doctor.

The orthopedic surgeon during telerehabilitation took significantly less time to consult patients (1.9 minutes, SD:0.5) than the traditional rehabilitation (15.2 minutes, SD:2.7). Patient satisfaction was higher for the telerehabilitation (78.3\%, SD:12.6) than for the orthopedic surgeon's traditional rehabilitation (36.7\%, SD:7.3) (Table 2).

Subjects reported a higher satisfaction with telerehabilitation than with the traditional orthopedic rehabilitation due to the fact that they spent less time at the hospital and had more time for exercises at home under orthopedic remote monitoring. It is assumed that less clinical time per subject using telerehabilitation translates into reduced costs of rehabilitation during the study period.

The telerehabilitation system can be used in complex rehabilitation of patients with injuries of the lower extremities. This will improve the quality of life in this group of patients and significantly reduce the cost of the rehabilitation period. These results provide preliminary evidence supporting the telerehabilitation model for orthopedic care. We conclude that telerehabilitation should be considered a key component in the long-term management of patients who have lower extremities injuries.

We are at the age of development of telemedicine, a technology that can exceed the capabilities of and can greatly enhance manual procedures and even existing technology. The simplicity of being able to apply this technology allowed it to be of great use in telerehabilitation and this paper shows importance of its implementation to the field of medicine.

\section{CONCLUSIONS}

An application of telemedicine is versatile process and it has endless possibilities of development, an application can mean accommodating more patients or discovering the best practice for a telerehabilitation, which will improve quality of patient's life. The implementation of these researches will be the most important contribution, which

Table 2. Comparison between visit time length and patient satisfaction for telerehabilitation $(n=32)$ and traditional orthopedic rehabilitation $(n=16)$

\begin{tabular}{ccccc} 
& Mean value for telerehabilitation (SD) & Mean value for traditional rehabilitation (SD) \\
\hline Visit time length, in minutes & 1.9 & 0.5 & 15.2 & 2.7 \\
\hline Patient satisfaction, \% & 78.3 & 12.6 & 36.7 & 7.3 \\
\hline
\end{tabular}


is why it is also important to begin researching on how this technology can be made more cost effective so it can be used in rural areas and underdeveloped hospital facilities.

\section{REFERENCES}

1. Baldwin K.D., Bernstein J., Ahn J. et al. Level of evidence gap in orthopedic research. Orthopedics. 2012;35(9):e1416-e1419. doi: 10.3928/01477447-20120822-31.

2. Bernstein J., Ahn J., Veillette C. The future of orthopaedic information management. J Bone Joint Surg Am. 2012; 94(13):e95.

3. Fayaz H.C., Jupiter J.B., Pape H.C. et al. Challenges and barriers to improving care of the musculoskeletal patient of the future-a debate article and global perspective. Patient Saf Surg. 2011;5:23. doi: 10.1186/1754-9493-5-23.

4. Kobbe P., Vodovotz Y., Kaczorowski D.J. et al. The role of fractureassociated soft tissue injury in the induction of systemic inflammation and remote organ dysfunction after bilateral femur fracture. Journal of Orthopaedic Trauma. 2008;22(6):385-90. 10.1097/ B0T.0b013e318175dd88

5. Dannecker K.L., Sazonova N.A., Melanson E.L. et al. A comparison of energy expenditure estimation of several physical activity monitors. Med Sci Sports Exerc 2013, 45(11):2105-2112.

6. Davies C.A., Spence J.C., Vandelanotte C. et al. Meta-analysis of internetdelivered interventions to increase physical activity levels. Int J Behav Nutr Phys Act. 2012; 9:52.

7. Tsvyakh A., Hospodarskyy A. Telerehabilitation of patients with injuries of the lower extremities. Telemed J E Health. 2017; 23: 1011-1015. https://doi.org/10.1089/tmj.2016.0267.

8. Binkley J.M., Stratford P.W., Lott S.A. et al. The Lower Extremity Functional Scale (LEFS): scale development, measurement properties, and clinical application. North American Orthopaedic Rehabilitation Research Network. Phys Ther. 1999; 79(4):371-83.

9. Tudor-Locke C., Ainsworth B.E., Thompson R.W. et al. Comparison of pedometer and accelerometer measures of free-living physical activity. Med Sci Sports Exerc. 2002; 34(12):2045-2051.

10. Bassett D.R., Mahar M.T., Rowe D.A. et al. Walking and measurement. Med Sci Sports Exerc 2008; 40(7):529-536.

11. Welk G.J., McClain J., Ainsworth B.E. Protocols for evaluating equivalency of accelerometry-based activity monitors. Med Sci Sports Exerc 2012; 44(1):39-49.

12. De Cocker K., Cardon G., De Bourdeaudhuij I. Validity of the inexpensive Stepping Meter in counting steps in free living conditions: a pilot study. Br J Sports Med. 2006; 40(8):714-716.

\section{Funding:}

This work was funded by the Ministry of Health,Ukraine, № 0119 U000608.

Research Topic: «Development of specialized medical equipment and treatment and rehabilitation techniques for providing telemedicine (remote) care to patients with injuries and diseases of the musculoskeletal system».

\section{ORCID and contributionship:}

Andriy I. Tsvyakh: 0000-0003-0884-4024 ${ }^{F}$

Andriy Ya. Hospodarskyy: 0000-0002-9394-2675 ${ }^{D}$

Nataliia O. Marchenkova: 0000-0002-3021-8882 ${ }^{A}$

Ihor R. Kopytchak: 0000-0002-3400-8823 ${ }^{E}$

Volodymyr P. Kostjuk: 0000-0001-6733-6041 ${ }^{B}$

Yevhen A. Lymar: 0000-0001-9428-5374 ${ }^{\mathrm{C}}$

Sergii M. Gdanskyi: 0000-0001-7289-1154 ${ }^{\mathrm{C}}$

\section{Conflict of interest:}

The Authors declare no conflict of interest.

\section{CORRESPONDING AUTHOR Nataliia 0. Marchenkova \\ Horbachevskyy Ternopil National Medical University \\ 1 Majdan Voli st., 46001 Ternopil, Ukraine \\ tel:095 1019939 \\ e-mail: marchenkovano@tdmu.edu.ua}

Received: 11.03 .2020

Accepted: 20.10 .2020

A - Work concept and design, B - Data collection and analysis, C - Responsibility for statistical analysis, D-Writing the article, $\mathbf{E}-$ Critical review, $\mathbf{F}-$ Final approval of the article 